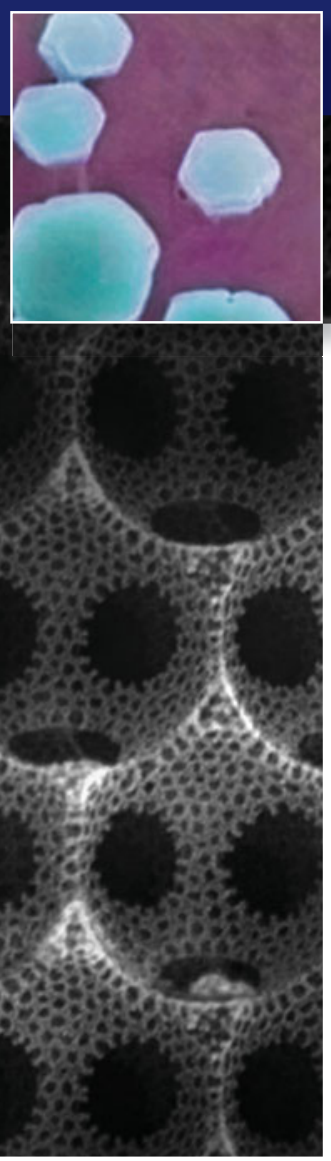

\title{
Hierarchical materials: Background and perspectives
}

\author{
Leon Mishnaevsky Jr. and Michael Tsapatsis, Guest Editors
}

\begin{abstract}
Hierarchical design draws inspiration from analysis of biological materials and has opened new possibilities for enhancing performance and enabling new functionalities and extraordinary properties. With the development of nanotechnology, the necessary technological requirements for the manufacturing of hierarchical materials are advancing at a fast pace, opening new challenges and opportunities. This article presents an overview of possible applications of and perspectives on hierarchical materials.
\end{abstract}

\section{Hierarchical structures of materials: Origins of the idea}

Starting from steel swords with complex structures to mud and straw-based buildings in Mali to ancient mortars and concrete, efforts to enhance the properties of materials by changing their structures have always been at the center of technological development. Composites, materials consisting of two or more dissimilar constituents with different properties, emerged from such efforts. While the possibilities of composite materials seemed infinite at first, two observations came into focus: (1) the composite constituents have their own, sometimes complex, structures that can in turn be modified, and (2) the potential for improving the properties of composite materials by adding or rearranging reinforcements is often limited - while some properties (e.g., stiffness) can be improved by increasing the volume content of hard reinforcements, other properties (e.g., fracture toughness) degrade. This motivated modifications of structure and properties of composite constituents and their control at several scale levels. ${ }^{1}$

At the same time, the extraordinary properties of materials found in nature, including wood, nacre, bone, and other biological materials, attracted the interest of the scientific community. It was observed that one of the main sources of such extraordinary properties of biocomposites is their complex hierarchical structure. In his classic paper, Lakes ${ }^{2}$ summarized the main ideas of hierarchical materials as a "basis for synthesizing new microstructures, which give rise to enhanced or useful physical properties." This provided the impetus for the development of new, bioinspired materials based on biomimicry principles. ${ }^{3}$ With the continuing advances and development of nanotechnology, new possibilities emerged to enable the manufacture of hierarchical materials with constituents modified on the nanoscale.

\section{Biological hierarchical materials: Variety of structures and biomimicking}

Natural biological materials often demonstrate extraordinary strength, damage resistance, and hardness. For instance, nacreous mollusk shells, which consist of $95 \% \mathrm{CaCO}_{3}$ by volume, have double the strength and exhibit a work of fracture that is 3000 times higher than that of monolithic $\mathrm{CaCO}_{3}{ }^{4}$ Numerous studies have been devoted to the analysis of the sources of such extraordinary properties of biological materials. Several features have been identified, including the staggered brick-and-mortar structure and interlocked platelets of nacre (see Figure 1),$^{5-8}$ the layered structure with randomly distributed layer thicknesses found in the spicules that provide structural support in certain sea sponges, the functionally graded structures (graded distributions of reinforcement) of bamboo ${ }^{9}$ and tooth, ${ }^{10}$ and features of wood such as a cellular multilayered structure and fiber/fibrils with varied distribution 


\section{HIERARCHICAL MATERIALS: BACKGROUND AND PERSPECTIVES}

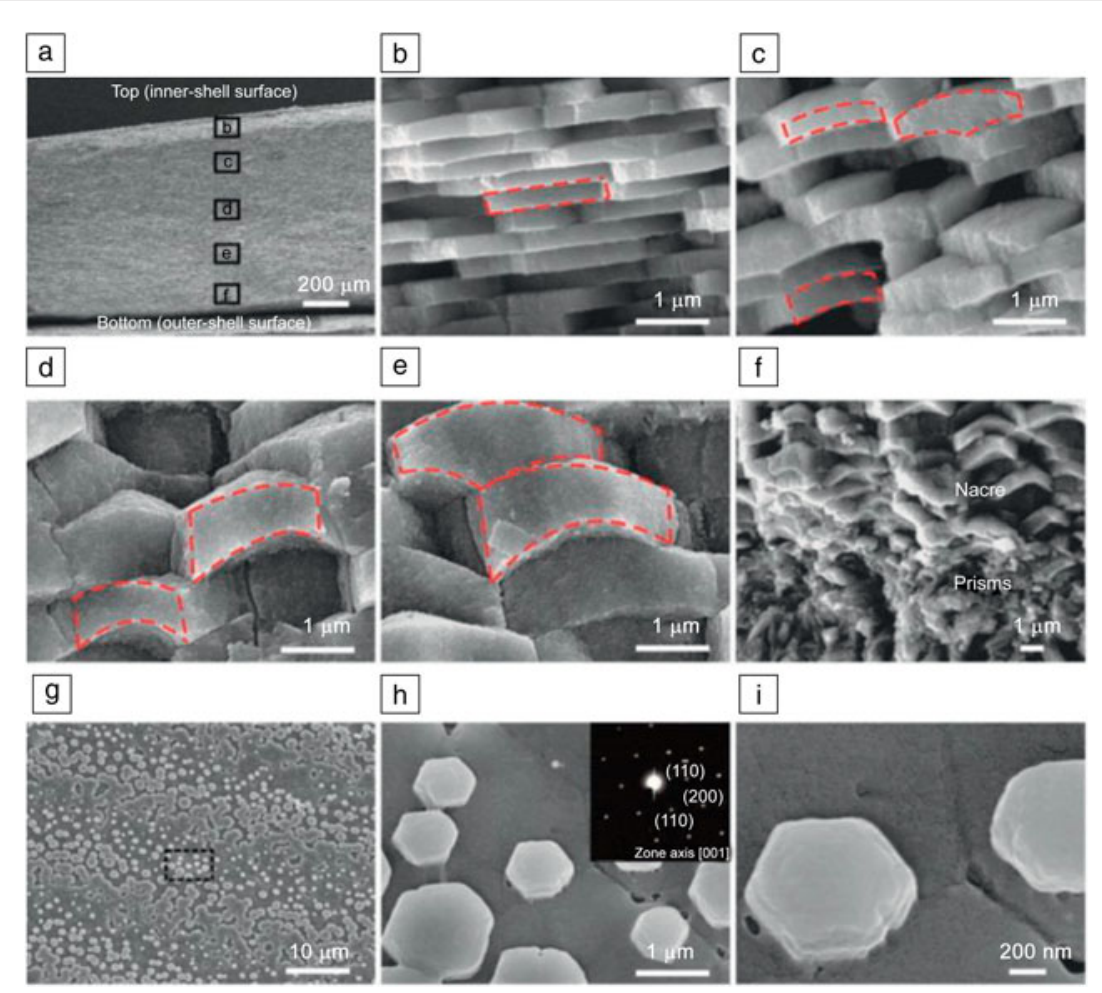

Figure 1. Nacre at nanoscale level-scanning electron micrographs showing crosssectional views and a plane view at the shell center. (a) Full view of a cross-sectional slice. (b-f) Detailed views of the corresponding marked areas in (a). (g) Full view of the inner surface at the shell center. (h) Detailed view of the labeled area in (g). (i) Enlarged view of (h). The areas marked in (b-e) highlight some typical platelet shapes. Reprinted with permission from Reference 8. (C) 2015 Elsevier.

hierarchical surface topography (e.g., adhesives inspired by gecko feet), and antifouling and self-cleaning surfaces that mimic shark skin and lotus leaves. ${ }^{15}$

\section{Interface to biological tissues: Hierarchical materials for medical applications}

The biomimetic roots of hierarchical materials and their structural similarity to biological tissues suggested medical uses (implants, hardtissue engineering, and drug delivery) as one of their first application areas. Attributes required for materials used in medical implants include biocompatibility and mechanical properties similar to those of bone, such as porosity and osteoconductivity (the ability to provide templates for new bone formation). Several groups are developing hierarchical materials with structures similar to those of hard tissues, including 3D structures of inorganic mineral plates and organic matrices. ${ }^{16}$ Ceramic-based hierarchical composites with bioactive calcium phosphate derivatives and bioglass using organic synthetic polymers (collagen, celluloses, gelatin) have been developed for bone-tissue engineering applications ${ }^{17}$ Nano-hydroxyapatite collagen composites have structures similar to that of bone and show excellent bioactive and biodegradable properties. ${ }^{18}$ Mesoporous bioactive glasses produced by $3 \mathrm{D}$ printing with

and density. ${ }^{11}$ In most cases, biological materials are hierarchical materials, with varied but still complex (lamellar, cellular, staggered, porous) structures at multiple scale levels. In their article in this issue, Weinkamer and Fratzl provide a more detailed overview of the structures and properties of natural hierarchical materials.

The connection between the extraordinary performance of biological materials and their hierarchical structure motivated research toward new man-made materials using the same principles. ${ }^{12}$ Tough and strong alumina ceramic-based materials with a nacre-like brick-and-mortar structure have been made using freeze-casting of nanoparticle suspensions. ${ }^{13}$ Polyurethane-based composites for stretchable electronics were developed using solvent-based hierarchical reinforcement approaches, with nanoclay and inorganic particles distributed in a polyurethane matrix. ${ }^{14}$ Materials with hierarchical porous structures (such as sponges or bone) demonstrate high permeability and surface area and can be used in catalytic and gas-adsorption applications. Other applications of materials with hierarchical porosity are bone implants (improving osteointegration of implants, thanks to large pores) and materials for impact protection. Such materials can be produced by templating or three-dimensional (3D) printing. A special case of bioinspired hierarchical materials is materials with polymer addition have a hierarchical pore structure and demonstrate good mineralization ability as scaffolds for bone regeneration. ${ }^{19}$

For hierarchical drug-delivery materials, requirements include the ability for controlled drug release combined with mechanical strength and biodegradability. Hierarchical composites with multiscale porous architectures can meet these requirements. Hierarchical porous composites with $\mathrm{CaCO}_{3}$ and polymer hybrids obtained via self-assembly biomineralization have enabled controlled release for cancer treatment. ${ }^{20}$ The importance of hierarchical materials for medical applications is related to the fact that medical materials interact directly with the biological tissues and are subject to the same service conditions; similar requirements dictate similar structures in this case.

\section{Strong and tough materials: Enhancing performances beyond limits}

A typical example of conflicting materials properties is the desire for combined high strength and toughness - if a material is reinforced with stiff particles, its strength and stiffness tend to grow with increasing reinforcement content, but the toughness might be reduced. This challenge can be overcome by using hierarchical structures. Kanzaki et al. ${ }^{1}$ developed an 
alumina-based ceramic material within the framework of the Japanese "synergy ceramics projects" with both high strength and toughness, achieved by a combination of aligned anisotropic grains (at microlevel) with the intragranular dispersion of nanoparticles (at nanolevel).

Another example of managing conflicting or competing properties is that of unidirectional fiber-reinforced polymer composites. These lightweight and strong materials are used in wind-energy applications, ${ }^{21,22}$ structural engineering, and the automobile industry. While the fiber reinforcement ensures excellent tensile stiffness and strength of the composites, their fatigue resistance and compressive strength (important factors, among others, for wind-energy applications) need some improvements. The addition of a small amount of nanoparticles (carbon nanotubes, graphene, or nanoclay) in the epoxy polymer matrix or fiber/matrix interface (fiber coating, so-called sizing) of the composites allows their fatigue lifetime to be increased by several times (see Figure 2). ${ }^{23}$

Biosensors used to detect analytes in the body (e.g., blood glucose) rely on hierarchical polymer structures that ensure higher surface area and faster diffusion of molecules. ${ }^{24}$ For example, 3D hierarchically porous polyaniline hydrogels have been used in new enzyme-based glucose sensors. ${ }^{25}$ In their article, Gorbatikh et al. discuss in greater detail the applications of hierarchical structures for property enhancements of lightweight polymers. Sidorenko et al. further discuss the application and potential of hierarchical materials in machining technologies in their article in this issue.

\section{Hierarchical materials for energy storage and conversion, adsorption, and catalysis: Transport and diffusion properties}

Important areas of the application of hierarchical materials include energy storage and conversion. The electrochemical properties of materials can be greatly enhanced by using materials with hierarchical structures, which can provide larger
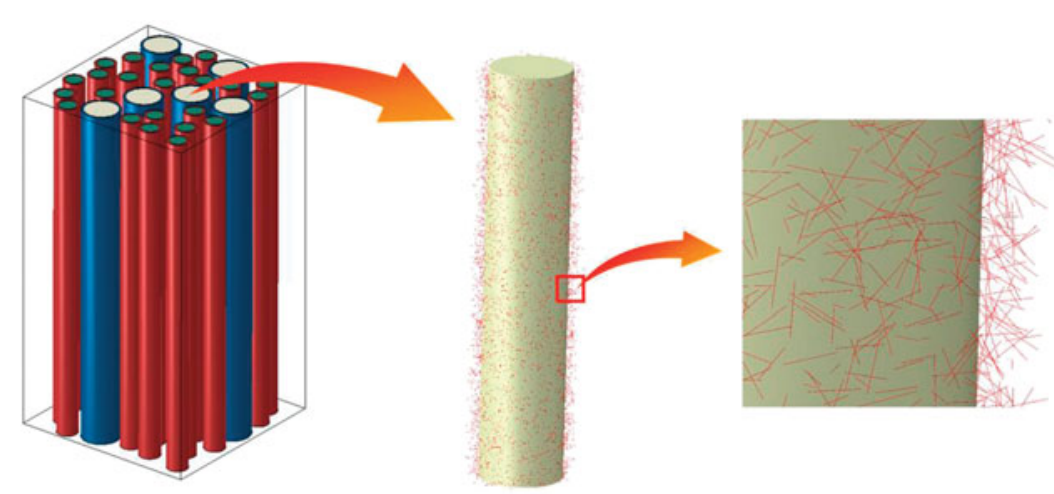

Hybrid FiberReinforced Composite
Fiber with CNT Reinforcements

\section{Detailed View}

Figure 2. 3D computational model of fiber-reinforced polymer composites with secondary nanoparticle reinforcement. Reprinted with permission from Reference 23. () 2014 Elsevier. Note: CNT, carbon nanotube. surface areas for interfacial diffusion, transport, and reactions. This opens new possibilities for the development of materials with improved electrical conductivity, facilitated diffusion paths, and electrolyte migration for applications such as electrode scaffolds and catalyst supports. ${ }^{26}$ Efforts have been made to develop hierarchical carbon-based nanocomposites for the electrodes of electrochemical supercapacitors. Due to their large surface area, open porosity, and high conductivity, these materials ensure improved energy densities for these devices. For lithium-ion batteries, using hierarchical materials as anodes allows reduction of the diffusion path of lithium ions and also accommodates volume changes due to alloying/ dealloying.

In fuel-cell applications, using hierarchical nanostructured carbon as the cathode catalyst in polymer electrolyte fuel cells achieves enhanced catalytic activity for oxygen reduction and improved overall cell performance, ${ }^{20}$ while hierarchically porous chitosan/graphene composites as anodes deliver good power densities. $^{27}$

For materials used as adsorbents and catalysts, hierarchical organization of porosity at the micro-, meso-, and macrolevel can impact performance characteristics such as activity, selectivity, and stability. ${ }^{28}$ In his article, Snyder discusses various synthesis strategies based on direct synthesis, post-synthetic modifications, and combinations thereof that are being developed. In their article, Valtchev and Mintova discuss hierarchical zeolites (ordered microporous materials incorporating meso- and macropores) that find uses in industrial applications such as crude oil cracking catalysts. ${ }^{26}$ A major motivation for hierarchical organization of porosity is to control molecular traffic in order to reduce diffusion limitations or improve selectivities. In addition, hierarchy in catalytic activity can be accomplished by selective placement of catalytic centers at distinct pore environments, enabling cascade reactions. ${ }^{29}$

\section{Computational modeling}

The main directions for computational modeling of hierarchical materials and structures include the analysis of interactions and synergies between scales (i.e., hierarchical load sharing), ${ }^{30}$ analysis of multiphysics and multiple functionality aspects of hierarchical materials, direct microstructure-based simulations, ${ }^{31}$ and analysis of nanoscale effects of materials behavior.

One approach to simulating the hierarchical structures of materials is based on hierarchical load-sharing models. According to this approach, the load is transferred from the largerscale structures ("roots") of the hierarchical "tree" to the lower "branches" and down to the smallest elements of the material (e.g., fibers, in the case of long fiber-reinforced composites). ${ }^{29}$ The load is shared equally among all of the 
sub-elements of a given branch (as long as they are intact) or among remaining intact sub-elements after some of them fail. Such models allow the effects of hierarchical structures and hierarchical load transfer to be analyzed in their pure form, as they only address the hierarchical structures.

Another more detailed modeling approach is based on direct numerical multiscale modeling of each structural level. In this case, wood is modeled as a cellular material, with multilayered walls and various nanoscale structures in each wall layer. ${ }^{11}$ Hierarchical nanoreinforced composites are also modeled as multilevel materials, with microscale and nanoscale reinforcements. ${ }^{22}$ The main computational challenges in such models are the large gap between scales, the high-aspect ratio of reinforcements (such as carbon nanotubes or graphene), and the strong nonlinearity of the material behavior, all requiring additional computational resources.

A review of the modeling techniques used for hierarchical materials is given in the article by Signetti et al. in this issue.

\section{Hierarchical materials: Summary and perspectives}

Materials with hierarchical structures represent a promising approach to enhance performance far beyond what can be achieved using composite structures, to add new functionalities and to adapt to special requirements (such as osteoconductivity in implantology).

The idea of mimicking biological materials provided the first impetus to the development of materials with hierarchical structures. Current directions for the development of hierarchical materials are more goal oriented. In many cases, structures at different scale levels play different, complementary roles, and ensure different functionalities for multifunctionality applications. Development of nanoscale technologies capable of delivering the required nanoscale structures (building blocks) and corresponding properties is a major prerequisite. Manufacturing by organizing nanoscale building blocks (polymer chains, macromolecules, nanoparticles) into structures is the main challenge for hierarchical materials development. A promising direction for overcoming this challenge is the spontaneous organization (self-assembly) of the building blocks into the required structures driven by physical or chemical mechanisms. ${ }^{32,33}$

The articles in this issue overview the state-of-the-art of aspects related to the analysis and development of hierarchical materials. Using biomaterials and multiscale modeling as starting points, we seek to enhance the performance and add new functionalities to hierarchical materials for lightweight structural and energy applications, catalysis, and machining of materials.

\section{References}

1. S. Kanzaki, M. Shimada, K. Komeya, A. Tsuge, Key Eng. Mater. 161-163, 437 (1999).

2. R. Lakes, Nature 361, 511 (1993).

3. P. Fratzl, M.J. Harrington, Introduction to Biological Materials Science (Wiley, New York, 2015).

4. P.R. Rao, Sādhanā 28 (3-4), 657 (2003).

5. M. Sarikaya, Proc. Natl. Acad. Sci. U.S.A. 96, 14183 (1999).

6. K.S. Katti, D.R. Katti, S.M. Pradhan, A. Bhosle, J. Mater. Res. 20 (5), 1097 (2005).

7. M.A. Meyers, P.Y. Chen, M.I. Lopez, Y. Seki, A. Lin, J. Mech. Behav. Biomed. Mater. 4 (5), 626 (2011).

8. J. Xu, G. Zhang, Mater. Sci. Eng. C 52, 186 (2015)

9. S. Amada, Y. Ichikawa, T. Munekata, Y. Nagase, H. Shimizu, Compos. Part B Eng. 28 (1-2), 13 (1997)

10. E.D. Yilmaz, G.A. Schneider, M.V. Swain, Philos. Trans. A Math. Phys. Eng. Sci. 373 (2038), 20140130 (2015).

11. H. Qing, L. Mishnaevsky Jr., Int. J. Solids Struct. 47 (9), 1253 (2010).

12. A.R. Studart, R.M. Erb, R. Libanori, in Hybrid and Hierarchical Composite Materials, C.-S. Kim, C. Randow, T. Sano, Eds. (Springer, New York, 2015), p. 287 13. F. Bouville, E. Maire, S. Meille, V.B. de Moortele, A.J. Stevenson, S. Deville, Nat. Mater. 13 (5), 508 (2014).

14. R. Libanori, F.H.L. Munch, D.M. Montenegro, A.R. Studart, Compos. Sci. Technol. 72 (3), 435 (2012)

15. K. Liu, L. Jiang, Annu. Rev. Mater. Res. 42, 231 (2012)

16. M. Kumar, K. Agrawal, D. Lahiri, in Hybrid and Hierarchical Composite Materials, C.-S. Kim, C. Randow, T. Sano, Eds. (Springer, New York, 2015), p. 203

17. K. Furuichi, Y. Oaki, H. Ichimiya, H. Imai, Sci. Technol. Adv. Mater. 7 (2), $219(2006)$

18. C. Du, F. Cui, X.D. Zhu, K. de Groot, J. Biomed. Mater. Res. 44, 407 (1999). 19. Y. Luo, C. Wu, A. Lode, M. Gelinsky, Biofabrication 5 (1), 015005 (2013). 20. J. Shi, W. Qi, C. Du, J. Shi, S. Cao, J. Appl. Polym. Sci. 129 (2), 577 (2013)

21. L. Mishnaevsky Jr., P. Brøndsted, R. Nijssen, D.J. Lekou, T.P. Philippidis, Wind Energy, 15 (1) 83 (2012).

22. L. Mishnaevsky Jr., Comput. Mech. 50 (2), 195 (2012).

23. L. Mishnaevsky Jr., G. Dai, Compos. Struct. 117, 156 (2014).

24. Y. Zhao, B. Liu, L. Pan, G. Yu, Energy Environ. Sci. 6, 2856 (2013).

25. L. Pan, G. Yub, D. Zhai, H. Ryoung Lee, W. Zhao, N. Liu, H. Wang, B.C.-K. Tee, Y. Shi, Y. Cui, Z. Bao, Proc. Natl. Acad. Sci. U.S.A. 109, 9287 (2012).

26. Y. Zhao, L. Peng, G. Yu, in Hybrid and Hierarchical Composite Materials, C.-S. Kim, C. Randow, T. Sano, Eds. (Springer, New York, 2015), p. 239

27. Z. He, J. Liu, Y. Qiao, C.M. Li, T.T.Y. Tan, Nano Lett. 12, 4738 (2012)

28. S. Mitchell, A.B. Pinar, J. Kenvin, P. Crivelli, J. Kärger, J. Pérez-Ramírez, Nat. Commun. 6, 8633 (2015).

29. C.M.A. Parlett, M.A. Isaacs, K. Beaumont, L.M. Bingham, N.S. Hondow, K. Wilson, A.F. Lee, Nat. Mater. 15, 178 (2016)

30. L. Mishnaevsky Jr., Compos. Sci. Technol. 71, 450 (2011).

31. L. Mishnaevsky Jr., Rev. Adv. Mater. Sci. 30, 60 (2012).

32. G.M. Whitesides, B. Grzybowski, Science 295 (5564), 2418 (2002)

33. G.M. Whitesides, M. Boncheva, Proc. Natl. Acad. Sci. U.S.A. 99 (8), 4769 (2002).

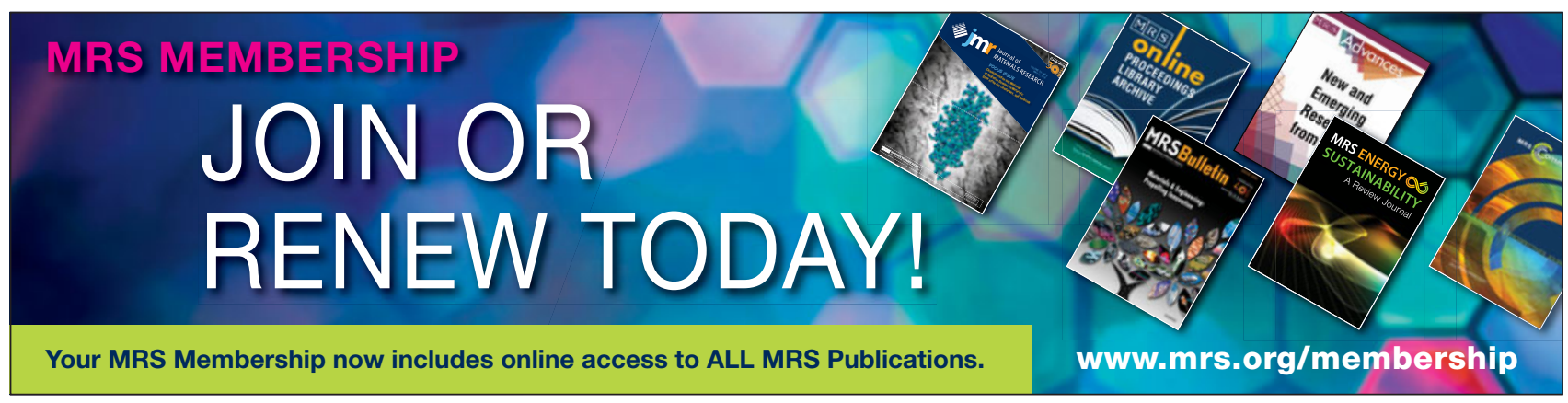

